\title{
The impact of COVID-19 on urologic practice, medical education, and training
}

\author{
Ananias C. Diokno ${ }^{1,2}$ (1) . Jeffrey M. Devries ${ }^{3}$
}

Received: 6 May 2020 / Accepted: 13 May 2020 / Published online: 28 May 2020

(c) Springer Nature B.V. 2020

Keywords Coronavirus · COVID-19 $\cdot$ Urologic practice $\cdot$ Medical training/education

\section{Introduction}

The COVID-19 virus has infected over 3.5 million people and killed more than 250,000 in at least 185 countries/sovereignties as of this writing. As a consequence of this disease that has overwhelmed health care facilities, and the lock-down order for citizens to stay home to prevent further spread of the disease, untold devastation to the economy of the world has unfolded. The devastating effects of this pandemic have significantly impacted every medical specialty. Our goal is to document the impact of the disease and the lock-down measure to urologic practice and practitionersat the level of attending physicians, residents, and medical students.

\section{Impact on urologic practice}

\section{Source of information}

In an effort to understand the impact of COVID-19 so far, the primary author did an informal survey of former urological residents who are now practicing in various sites in the United States of America. They were informed that their responses would be collected and reported in a de-identified manner. They were also asked to preview this paper and approve the content. Responses came from urologic

Ananias C. Diokno

ananias.diokno@beaumont.edu

1 Department of Urology, Oakland University William Beaumont School of Medicine (OUWBSOM), Rochester, MI, USA

2 University of Central Florida, Orlando, FL, USA

3 Graduate Medical Education, Department of Pediatrics, Beaumont Health, OUWBSOM, Rochester, MI, USA practices on the west coast (California), south (Texas), east coast (New Jersey), and midwest (Missouri, Iowa, Michigan, Ohio). The responses were remarkably distressing, and expressed significant uncertainty about the future.

\section{The essence of true partnership}

First, a personal heartwarming report came from a senior urologist in a small group practice in the midwest. The urologist was considered to be at high risk because of his age and co-morbid conditions. His partners "ordered" him quarantined at home and assigned him only limited video telemedicine and phone conferences, while they took care of any urgent and emergent cases in the hospital and clinic, and managed the day-to-day operations of their practice. Although he volunteered to relinquish his salary, his partners - to their great credit—-kept his salary fully in place.

\section{Impact on the outpatient setting}

The experience of all respondents regarding practice downsizing began abruptly in mid-March. Very quickly, all elective cases were cancelled, with no future specific date in mind. Interestingly, this timing was approximately 2 months behind the January 23 closure of all elective procedures in a large hospital in Wuhan, China, according to one urologist in that hospital. The first few days of practice lock-down involved hundreds of phone calls to cancel scheduled surgeries and office visits. They continued to see in their office all urgent and emergent cases that could be managed in the office, including catheterizations, cystoscopy, stent placements, cancer treatments, and pain mitigation. Most estimated that their outpatient office work was reduced by $40-80 \%$. They replaced many of the lost clinic visits with Telemedicine, which they estimated accounted for $25-80 \%$ of their office care at that time. 
Telemedicine practice ranged from phone to tablet to computer, using FaceTime ${ }^{\odot}$, Zoom $^{\odot}$, and other sophisticated software platforms. Both physicians and patients were challenged to successfully work on these platforms that were new to them, and those without full IT services at their disposal often called upon younger, more computersavvy family members for assistance! Unfortunately, at this point, there is no general consensus regarding which urologic conditions can safely and successfully be managed by telemedicine, nor is there either training or testing to assess and certify proficiency of the providers. Even the determination of reasonable reimbursement needs to be determined. One urologist expressed frustration caring for a young man with testicular pain in the absence of a physical exam. He acknowledged that it was suboptimal care, and wondered whether this would become part of the "new normal".

\section{Impact on support staff}

One of the significant issues expressed by the urologists was how to manage their offices' support staff. Respondents had significant differences, based partially on type of practice. A major urologic practice on the west coast has 50\% capitated practice and another $20 \% \mathrm{HMO}$ patients. They laid off only 2 of 24 staff members (who had planned to retire or leave the practice anyway), maintaining the others since their office finances were less significantly impacted. In a major urologic practice in the south, working for a large group, the administrators decided not to reduce their staff, despite a significant reduction of patient visits. In the midwest, one group furloughed $33 \%$ of its staff, another group 50\%, and one employed urologist was able to avoid lay-offs entirely.

\section{Impact on practice income and health status}

As one considers the changes that have occurred among these urologists, the impact of the lock-down on their practices is somewhat predictable. The group that seemed to fare the best is the California practice with significant capitated practice. He estimated that practice income will be reduced initially by $10-20 \%$, up to a maximum loss of $30-40 \%$ because of postponed elective surgeries. The remainder of respondents are anticipating an income loss of at least $50-80 \%$. Of all the respondents, the health care team members of only one practice group on the east coast were directly impacted by this disease. Of the two urologists and a support staff member who contracted COVID-19, two of the three had mild symptoms, while one urologist developed severe symptoms requiring hospitalization; he has subsequently recovered and returned to practice.

\section{Impact on medical training}

\section{Source of information}

I had the opportunity to interview the Director of Graduate Medical Education of one of the major teaching health systems in the Midwest USA. The following describes how residents and students have been managed to date there and elsewhere.

\section{Impact on medical students}

Medical students across all specialties were dismissed from the hospital. There was an initial discussion about incorporating them with the front-line health care workers, but this was not further considered because of the need for them to be more closely supervised, which would not have been possible in the current situation. Another reason was the limited supply of personal protective equipment (PPE), which needed to be preserved for more experienced providers. In fact, both to limit health care workers' exposure and to preserve PPE, medical teams were asked to limit the number of providers who actually had to enter the rooms of COVID patients. To the extent they were available, patients were provided with electronic tablets, thereby enabling them to communicate with their caregivers outside the room. Senior students who had met all requirements to graduate early were offered the opportunity to begin residency a few months ahead of time in some of the hospitals to which they had matched, but very few agreed to do so. Although their reasons are largely unknown, it is suspected that one of the reasons might be pressure exerted by their families who feared the consequences of this earlier-than-required exposure.

\section{Impact on residents and fellows}

Residents and fellows were re-deployed to areas in which their level of expertise could be utilized. Both common sense and the ACGME required that the residents and fellows receive adequate supervision in all cases (albeit sometimes by physicians from specialties different than their own), and that all standard duty hours restrictions be maintained. One might think that this latter requirement might be waived in such a crisis, but it was maintained precisely because of the requirement's initial intent-to reduce medical errors, to which providers might be particularly susceptible during such anxious circumstances. In most cases, residents who were immunosuppressed, pregnant, or at high risk because of co-morbidities were sent home, or to venues with minimal risk of exposure (e.g. providing telehealth visits). Examples 
of re-deployment include Dermatology trainees who were assigned to internal medicine COVID units, while senior internal medicine residents were assigned to the intensive care units (ICU), where their expertise could be maximally utilized. Surgical residents, including Urology residents, were assigned to medical units and intensive care units. Urology residents proved to be particularly valuable when dealing with difficult Foley insertions, or patients with organ failure who needed accurate fluid output monitoring. They continued to assist or perform emergency and some urgent surgical cases under the supervision of their attending surgeons, who were available to care for these types of cases.

In mid-March, the Accreditation Council for Graduate Medical Education (ACGME) designated three new categories of status related to Coronavirus, to which each hospital could self-declare for up to a renewable, 30-day period. These categories were "Stage 1: Business as Usual"; "Stage 2: Increased Clinical Demands"; and "Stage 3: Pandemic Emergency", in which the increase in volume and/or severity of illness creates an extraordinary circumstance in which standard educational requirements could be superseded by urgent and emergent patient care needs. Declaration of a particular stage required agreement between the Director of Graduate Medical Education and the hospital's clinical leadership. Under Stage 3, most ACGME institution- and program-specific rules were temporarily suspended, with the exception of the need for adequate supervision, maintenance of duty hour restrictions, and the necessity to protect the health care team with adequate PPE. It was the lifting of requirements under Stage 3 that permitted the re-deployment of residents and fellows, sometimes to other services and to other hospitals within the Sponsoring Institution.

\section{Impact on emergency services}

As the hospital focused on managing the pandemic, dealing mainly with the COVID patients and other emergent medical and surgical cases, there was massive "hemorrhage" of cash in the hundreds of millions of dollars. As a consequence, many hospitals had to temporarily lay-off workers, and, in some cases, permanently terminate positions. There were even a small number of physicians who were temporarily laid-off because of reduction of demand for their services. In a somewhat unsuspected but understandable phenomenon, it was noted that there were significant reductions in the number of myocardial infarction patients, trauma cases, and pediatric emergencies coming to Emergency Rooms compared to pre-COVID levels, presumably due to patient reluctance to seek care in an emergency room and risk COVID exposure. A consequent increase in deaths at home of individuals who needed care in Emergency Rooms but declined to seek it is suspected, but data are not available at this time. The reduction of ER visits was at least partially offset by a significant increase in telemedicine appointments, the development of which accelerated substantially during this period.

\section{Impact on physical and mental health}

COVID-19 has taken a toll on the physical and mental health of health care team members at all levels. Several residents and fellows were proven or suspected to be COVID positive. Across the country, multiple health care workers have contracted COVID, with deaths impacting nearly all levels of front-line providers. Since most trainees are relatively young and presumably without co-morbidities, the rate of more serious disease in this group has been low. Tragically, however, there have been COVID-related deaths of residents and faculty members. There has also been great anxiety caused by the fear of spreading COVID to family members. For that reason, several hotels and college dormitories-now emptied because of COVID-related stay-at-home ordershave been made available for health care workers, to separate them from their COVID-susceptible family members, thereby reducing at least one source of anxiety.

One major concern among the trainees and, for that matter, for all health care providers is their level of mental/emotional health. In the midst of the war, one focuses on the job to be done... and surviving. The continuing calls for CPR, the higher-than-usual proportion that are unsuccessful, and being surrounded by very sick and dying patients who cannot be surrounded by their own loved ones are major stressors for all providers. Hospitals have established Wellness resources available for both one-to-one consultation and group (virtual) face-to-face call-in sessions to be able to share stories and support. It is very much expected that once the acute surge subsides and everyone has more time to reflect, Post-Traumatic Stress Syndrome (PTSD) may continue for a long time to come. Equally disturbing is the possibility that the "Post-" in PTSD will actually become Persistent-Traumatic Stress Disorder, inasmuch as various models project a secondary surge of cases once geographic isolation mandates are lifted, possible persistence of disease over the summer, and possibly a new, seasonal upsurge in the fall.

\section{What does the future hold for medical practice and training?}

Of course, only time will tell. At this time, it is impossible to know even when that "post-COVID" future will start. We hope that the anticipated surges will be easily controlled by applying lessons learned and by the appropriate use of the newly developed therapeutics against COVID-19. We are also hoping that an effective vaccine will become available soon and be available for widespread distribution. These schemes if realized soon will reduce the need for hospital 
services including the use of intensive care units and respirators. One can only hope that-in public health and as a society - we have learned from this experience and will take the precautionary measures now to ensure that we will be better prepared to have a more rapid and effective response to future pandemics.

It is likely that the return to a non-crisis state will be gradual, both as different nations and states relax their restrictions at different rates, and as individuals who are more and less risk averse choose to manifest these traits in different ways and at different paces. Some of our experiences and lessons learned are likely to result in ongoing changes, such as increased reliance on telehealth visits. The strongly negative financial impact on health care systems, which may not end in the near future, will have its own impact-possibly for years to come.

During this crisis, health care workers have been widely and quite appropriately recognized as heroes. Let us leverage that good will as we plan for the next months of dealing with this crisis, and preparing adequately for the next.
Acknowledgements We like to recognize the contributions of the following urologists in sharing their experience during this initial period of the COVID-19 pandemic: Andrew Bernstein, Jeffrey Carey, Andrew Chung, Damon Dyche, Michael Ingber, Gregory McLennan, Aaron Nguyen. Joseph Salisz, and Allan Van Horn

Funding None.

\section{Compliance with ethical standards}

Conflict of interest The authors declare that they have no conflict of interest.

Publisher's Note Springer Nature remains neutral with regard to jurisdictional claims in published maps and institutional affiliations. 